\title{
THE EFFECT OF INITIAL DEFORMATIONS ON THE BEHAVIOUR OF A CYLINDRICAL SHELL UNDER AXIAL COMPRESSION*
}

\author{
BY \\ P. CICALA \\ Escuela Superior de Aerotécnica and University of Cordoba, Argentina
}

The theoretical investigation presented here concerns the effect of certain initial deformations on the buckling under axial compression of thin plate structures. A general theory is developed and used to make approximate analyses of the complete cylinder and curved panel. Two extreme cases of the curved panel are treated: (a) uniform longitudinal shortening and, (b) uniform longitudinal stress. Particular attention is paid to the possibility of inducing buckling in a favorable mode, and thus increasing the load which a structure can withstand.

\section{INTRODUCTION}

The buckling of a cylindrical panel under axial compression has recently been the subject of a number of investigations using the non-linear theory of thin plates. This theory is valid for deflections of the same order of magnitude as the thickness of the panel.

The non-linear theory has shown that, for a panel with perfect initial form and starting from the configuration of infinitesimal deflections which correspond to the critical loads of the linear theory, buckling may evolve through one of several paths. While some of these paths are favorable and give loads which increase with increasing deformations, others cause the load to fall after buckling. This has been proved quantitatively by von Kármán and Tsien ${ }^{1}(1)$ and, independently, also by the author (2).

Experiments have shown that the effect of initial deformations is usually to cause buckling in an unfavorable mode. However, Welter (3) has shown from compression tests on curved panels, that it is possible to delay buckling by initially bending a panel to a smaller radius than that at which it is tested. These results suggested the present investigation.

The General Equations for a Cylindrical Shell with Initial Deformations Let $t^{\prime}$ be the thickness of the shell and write $t=t^{\prime} / \sqrt{12\left(1-\nu^{2}\right)}$ where $\nu$ is Poisson's ratio. We call state " 0 " a state of deformation by which the mean surface takes the form of a circular cylinder whose radius is $R$; let $x$ and $y$ be the axial and circumferential coordinates, $w$ the radial displacements (positive outwards), leading from state " 0 " to the actual state " $A$ ".

Assuming that $t^{\prime}$ is small compared with $R$ and that the displacements $w$ have the same order of magnitude as $t^{\prime}$, the normal and shearing stresses in state " $\mathrm{A}$ " may be represented in the forms

$$
\sigma_{x}=E \frac{\partial^{2} f}{\partial y^{2}}, \quad \sigma_{\nu}=E \frac{\partial^{2} f}{\partial x^{2}}, \quad \tau=-E \frac{\partial^{2} f}{\partial x \partial y},
$$

*Received August 28, 1950.

${ }^{1}$ Numbers in parentheses refer to Bibliography at the end of the paper. 
where $E$ is Young's modulus and $f$ is a stress function. Similarly the stresses in state " 0 " can be derived from another stress function $f^{\prime}$. If the material is completely elastic, the stress variation from state " 0 " to state " $\mathrm{A}$ " is related to stretching by the equation

$$
\nabla^{4}\left(f-f^{\prime}\right)+k=0
$$

where

$$
k=\frac{\partial^{2} w}{\partial x^{2}}\left(\frac{\partial^{2} w}{\partial y^{2}}-\frac{1}{R}\right)-\left(\frac{\partial^{2} f}{\partial x \partial y}\right)^{2}
$$

The shell is held in state " 0 " by applying a certain distribution of normal pressure $E t^{\prime} p_{0}$, where $p_{0}$ is, in general, a funnction of $x$ and $y$; the quantity

$$
p^{\prime}=p_{0}-\frac{1}{R} \frac{\partial^{2} f^{\prime}}{\partial x^{2}}
$$

will be zero if the shell is free from transverse shearing stresses in state " 0 ". In general, this will not be the case; the quantity $E t^{\prime} p^{\prime}$ represents a net inherent pressure that adds to the plate and membrane pressures in any state of the shell. Therefore, the equilibrium condition in state "A", under the applied pressure $E t^{\prime} p$ yields

$$
p=p^{\prime}+t^{2} \nabla^{4} w-\frac{\partial^{2} w}{\partial x^{2}} \frac{\partial^{2} f}{\partial y^{2}}-\left(\frac{\partial^{2} w}{\partial y^{2}}-\frac{1}{R}\right) \frac{\partial^{2} f}{\partial x^{2}}+2 \frac{\partial^{2} w}{\partial x \partial y} \frac{\partial^{2} f}{\partial x \partial y} .
$$

If the shell is free from stress in state " 0 ", the functions $f^{\prime}$ and $p^{\prime}$ are both zero and Eqs. (2) and (4) degenerate to the known equations for a shell having perfect initial form and no initial stresses.

If a particular state $w=w^{*}$ exists for which the shell is free from stress when there is no normal pressure, we may write

$$
f^{\prime}=k^{*}, \quad p^{\prime}=-t^{2} \nabla^{4} w^{*}
$$

where $k^{*}$ is the value for $k$ when $w=w^{*}$. Eqs (2) and (4) then take the respective forms

$$
\begin{gathered}
\nabla^{4} f+k-k^{*}=0 \\
p=t^{2} \nabla^{4}\left(w-w^{*}\right)-\frac{\partial^{2} w}{\partial x^{2}} \frac{\partial^{2} f}{\partial y^{2}}-\left(\frac{\partial^{2} w}{\partial y^{2}}-\frac{1}{R}\right) \frac{\partial^{2} f}{\partial x^{2}}+2 \frac{\partial^{2} w}{\partial x \partial y} \frac{\partial^{2} f}{\partial x \partial y} .
\end{gathered}
$$

These equations have already been presented by the writer (2). They have been used by Dei Poli (4) in his studies on the cylindrical panel with initial deformations.

\section{The Complete Cylindrical Shell with Initial Deformations}

We consider the case of a cylindrical shell with the particular deformations represented by

$$
\begin{aligned}
& f^{\prime}=0 \\
& p^{\prime}=p_{1} \sin \xi \sin \varphi+p_{2} \cos 2 \xi+p_{3} \cos 2 \varphi+p_{4} \sin 3 \xi \sin \varphi
\end{aligned}
$$

with $\xi=\pi x / a, \varphi=\pi y / b$, where $a$ and $b$ are constants and $\pi R / b$ is any integer. The first of these equations states that the shell is free from stress in state " 0 ". The con- 
strained sections of the shell are assumed to be so remotely situated that their influence on the buckling process may be neglected.

To obtain an approximate solution to the problem of the shell under axial load, we first express the functions $f$ and $w$ in the following non-dimensional forms

$$
\begin{aligned}
& f / t^{2}=f_{1} \sin \xi \sin \varphi+f_{2} \cos 2 \xi+f_{3} \cos 2 \varphi+f_{4} \sin 3 \xi \sin \varphi-\eta y^{2} / R t \\
& w / t=w_{1} \sin \xi \sin \varphi+w_{2} \cos 2 \xi+w_{3} \cos 2 \varphi+w_{4} \sin 3 \xi \sin \varphi+w_{0} .
\end{aligned}
$$

The coefficient $\eta$, which is positive for compressive axial loads, represents the ratio of the mean axial stress to the critical stress $\sigma_{c}=-2 E t / R$ derived from the linear theory. The last term in the first of Eqs. (7) therefore relates to the mean axial stress.

To determine the values of the constants $f_{1}, f_{2}, f_{3}, f_{4}, w_{1}, w_{2}, w_{3}$ and $w_{4}$, we use the Galerkin method and evaluate the integral expressions

$$
\int_{0}^{2 a} d x \int_{0}^{2 b}\left[\nabla^{4} f+k\right] \psi_{1} d y=\int_{0}^{2 a} d x \int_{0}^{2 b} p \psi_{2} d y=0
$$

where for $\psi_{1}$ and $\psi_{2}$ we replace successively the functions

$$
\sin \xi \sin \varphi ; \quad \cos 2 \xi ; \quad \cos 2 \varphi ; \quad \sin 3 \xi \sin \varphi
$$

In this way we obtain

$$
\begin{aligned}
& D_{1} f_{1}=-\beta^{2} w_{1}+2 w_{1} w_{2}+2 w_{1} w_{3}-2 w_{2} w_{4}, \\
& 32 \beta^{2} f_{2} / \alpha^{2}=-8 \beta^{2} w_{2}+w_{1}^{2}-2 w_{1} w_{4}, \\
& 32 \alpha^{2} f_{3} / \beta^{2}=w_{1}^{2}+9 w_{4}^{2}, \\
& D_{4} f_{4}=-9 \beta^{2} w_{4}-2 w_{1} w_{2}+18 w_{3} w_{4}, \\
& D_{1} w_{1}=2 \eta \beta^{2} w_{1}+\beta^{2} f_{1}-2 w_{1} f_{2}-2 w_{2} f_{1}-2 w_{1} f_{3}-2 w_{3} f_{1}+2 w_{2} f_{4}+2 w_{4} f_{2}+c_{1}, \\
& 16 \beta^{2} w_{2} / \alpha^{2}=8 \eta \beta^{2} w_{2}+4 \beta^{2} f_{2}-w_{1} f_{1}+w_{1} f_{4}+w_{4} f_{1}+c_{2}, \\
& 16 \alpha^{2} w_{3} / \beta^{2}=-w_{1} f_{1}-9 w_{4} f_{4}+c_{3}, \\
& D_{4} w_{4}=18 \eta \beta^{2} w_{4}+9 \beta^{2} f_{4}+2 w_{1} f_{2}+2 w_{2} f_{1}-18 w_{3} f_{4}-18 w_{4} f_{3}+c_{4},
\end{aligned}
$$

where

$$
\begin{array}{rlrl}
D_{1} & =\left(\frac{\alpha}{\beta}+\frac{\beta}{\alpha}\right)^{2}, & D_{4}=\left(\frac{\alpha}{3 \beta}+\frac{3 \beta}{\alpha}\right)^{2}, \\
\alpha & =a / \pi \sqrt{R t}, & \beta & =b / \pi \sqrt{R t}, \\
c_{i} & =a^{2} b^{2} p_{i} / \pi^{4} t^{3}, & & (i=1,2,3,4) .
\end{array}
$$

Taking into account the equation

$$
\frac{\partial u}{\partial x}+\frac{1}{2}\left(\frac{\partial w}{\partial x}\right)^{2}=\frac{1}{E}\left(\sigma_{x}-\nu \sigma_{y}\right)=\frac{\partial^{2} f}{\partial y^{2}}-\nu \frac{\partial^{2} f}{\partial x^{2}}
$$


where $u$ is the axial component of displacement, the mean axial negative shortening

$$
\epsilon_{x}=\frac{1}{2 a} \int_{0}^{2 a} \frac{\partial u}{\partial x} d x
$$

may be calculated. The condition of uniform shortening, requiring that $\epsilon_{x}$ be independent of $\varphi$, is satisfied by Eqs. (7), when Eq. (11) is considered. Denoting by $\epsilon$ the constant value of $\epsilon_{x}$, we obtain

$$
-\epsilon R / t=2 \eta+\left(w_{1}^{2}+8 w_{2}^{2}+9 w_{4}^{2}\right) / 8 \alpha^{2} .
$$

For a given initial deformation and given wave length, the constants $c_{i}$ are first calculated from Eqs. (9)-(16) by replacing for the $w^{\prime}$ s the values $w_{1}^{\prime}, w_{2}^{\prime}, w_{3}^{\prime}, w_{4}^{\prime}$ that

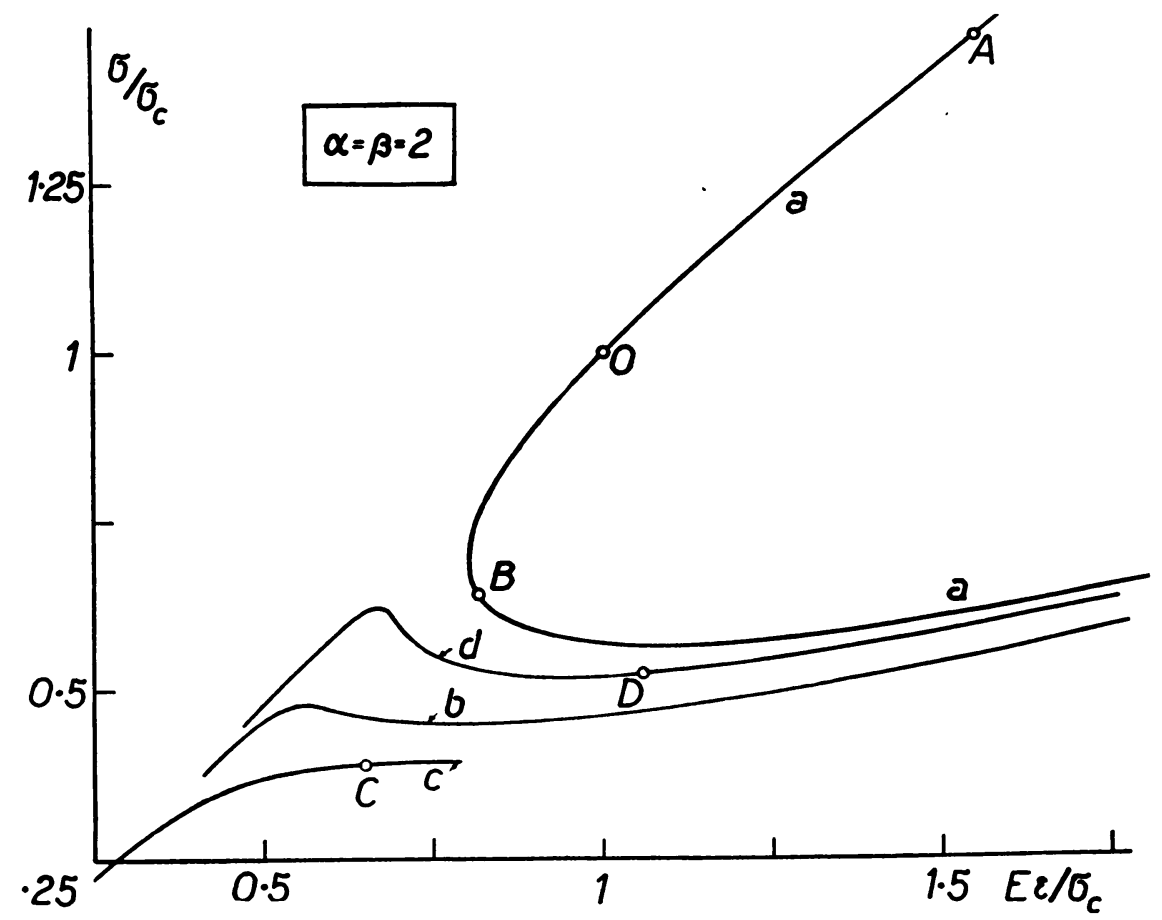

FIG. 1. Stress-strain diagram for the complete cylinder.

Line a $\left(w_{1}^{\prime}=w_{2}^{\prime}=0\right)$. Line $\mathrm{b}\left(w_{1}^{\prime}=w_{2}^{\prime}=1 / 4\right)$. Line c $\left(w_{1}^{\prime}=2, w_{2}^{\prime}=-1 / 4\right)$.

Line $\mathrm{d}\left(w_{1}^{\prime}=1 / 2, w_{2}^{\prime}=-1 / 16\right)$.

correspond to $\eta=0$. Then successive sets of values of $\eta, f_{1}, f_{2}, f_{3}, f_{4}, w_{1}, w_{2}, w_{3}, w_{4}$ satisfying Eqs. (9)-(16) are to be found. The corresponding stress, waveform and shortening can be deduced from Eqs. (7) and (19).

(a) First form of deformation equation

As a first step, we consider the case where

$$
\alpha=\beta, \quad w_{3}=w_{4}=f_{4}=0
$$

and hence disregard Eqs. (12), (15) and (16). In this case, the results obtained for a 
special value of $\beta$ may be generalized by considering that the parameter $\beta$ disappears from the equations if the quantities

$$
f_{1} / \beta^{4}, f_{2} / \beta^{4}, f_{3} / \beta^{4}, w_{1} / \beta^{2}, w_{2} / \beta^{2}, c_{1} / \beta^{6}, c_{2} / \beta^{6},\left(\eta / \beta^{2}\right)-\left(2 / \beta^{4}\right)
$$

are introduced.

The results of the calculations for $\beta=2$ are represented in Figs. 1 and 2. In Fig. 1, values of $\eta=\sigma / \sigma_{c}$ are plotted against $E \epsilon / \sigma_{c}$ for various values of $w_{1}^{\prime}$ and $w_{2}^{\prime}$. Considering

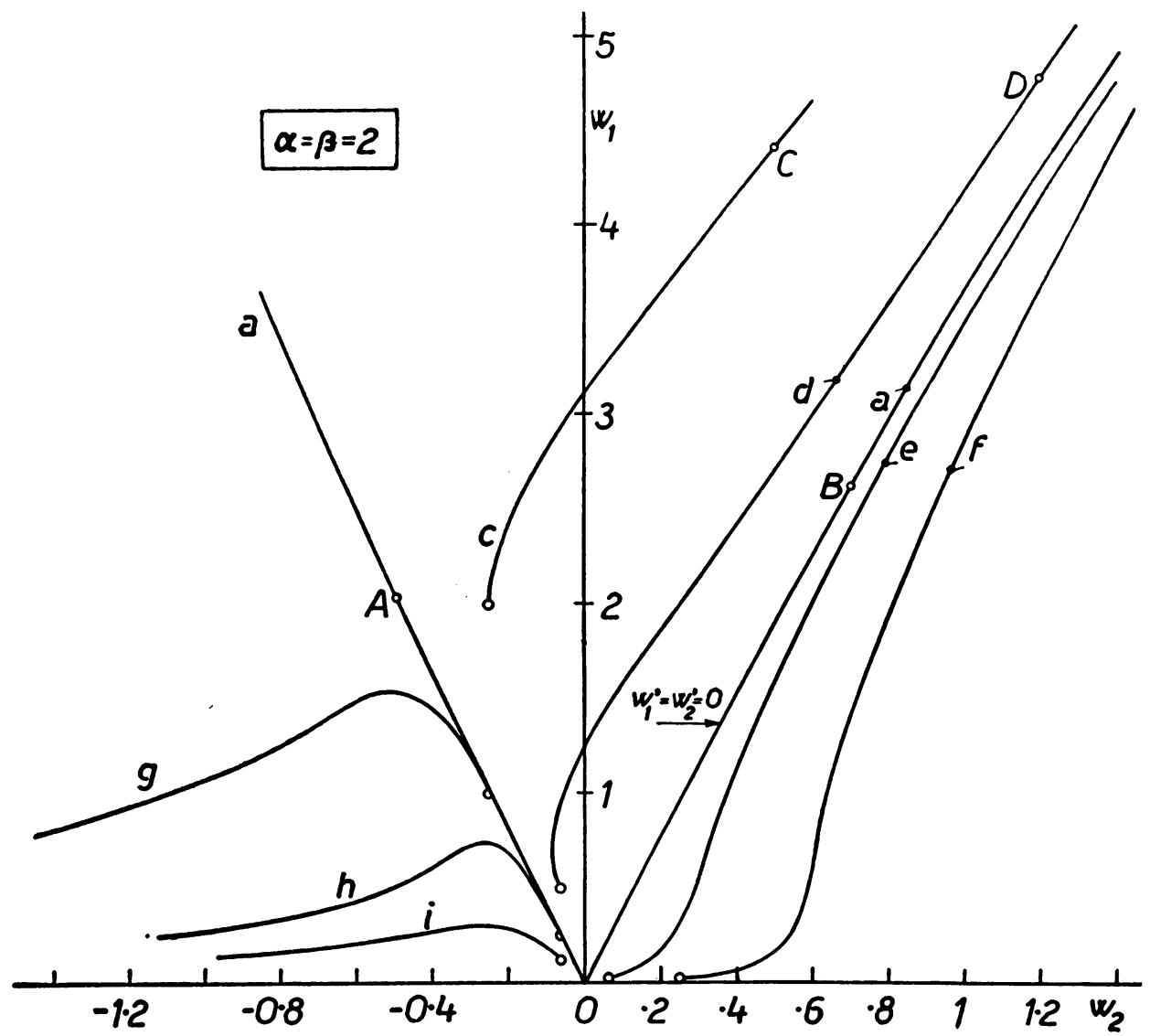

Fig. 2. Displacement components $w_{1}$ versus $w_{2}$.

Line a $\left(w_{1}^{\prime}=w_{2}^{\prime}=0\right)$. Line $\mathrm{c}\left(w_{1}^{\prime}=2, w_{2}^{\prime}=-1 / 4\right)$. Line $\mathrm{d}\left(w_{1}^{\prime}=1 / 2, w_{2}^{\prime}=-1 / 16\right)$.

Line e $\left(w_{1}^{\prime}=1 / 32, w_{2}^{\prime}=1 / 16\right)$. Line $\mathrm{f}\left(w_{1}^{\prime}=1 / 32, w_{2}^{\prime}=1 / 4\right)$.

Line $\mathrm{g}\left(w_{1}^{\prime}=1, w_{2}^{\prime}=-1 / 4\right)$. Line $\mathrm{h}\left(w_{1}^{\prime}=1 / 4, w_{2}^{\prime}=-1 / 16\right)$.

Line i $\left(w_{1}^{\prime}=1 / 8, w_{2}^{\prime}=-1 / 16\right)$.

the graph for a shell of perfect initial form $\left(w_{1}^{\prime}=w_{2}^{\prime}=0\right), O$ is the point at which buckling begins: for this point, $w_{1}=w_{2}=0$ and $\eta=1$. The lower branch of the curve through $B$ represents the unfavorable path with decreasing stresses and strains accompanying the first phase of increasing deformations. The upper branch of the curve through $\mathbf{A}$ represents the favorable path. If Hooke's law were obeyed and the curve followed the upper branch, the behaviour of the shell would be practically unaffected by buckling. 
Figure 2 shows graphs of the deflection components $w_{1}$ versus $w_{2}$ for various initial values $w_{1}^{\prime}$ and $w_{2}^{\prime}$. These graphs are limited to positive values of $w_{1}$, since the diagrams would be symmetrical with respect to the $w_{2}$ axis. The graph for a shell with $w_{1}^{\prime}=w_{2}^{\prime}=0$, indicates that, for small values of $w_{1}$ and $w_{2}, w_{1} \approx \pm 4 w_{2}$; the positive sign corresponds to the unfavorable branch of the curve in Fig. 1. A third possible state of equilibrium would be represented in Fig. 1 by a horizontal line through 0 ; this corresponds to axially symmetrical buckling with $w_{1}=0$.

From Fig. 2, the evolution of the buckled form of the cylinder may be traced for various initial deformations, the corresponding stress-strain diagrams being given in Figs. 1, 3 and $5^{2}$. The graphs of $w_{1}$ vs. $w_{2}$ in Fig. 2 are of two kinds: (a) those which approach that branch of the line for a cylinder of perfect initial form which has positive slope and, (b) those which tend to approach the $-w_{2}$ axis; in any case they diverge from line 0A. From Figs. 1, 3 and 5, it may be seen that the first kind of initial deformation leads to an unfavorable path while the second kind indicates a rather favorable behaviour with the load approaching the critical load of the linear theory. It will be seen later that this latter possibility is fictitious.

(b) Second type of deflection equation

Calculations taking into account the $w_{3}$ component of displacement and neglecting $w_{4}$ and $f_{4}$ as before, have already been carried out (2) for the case of a cylindrical shell with perfect initial form-that is, with $c_{1}=c_{2}=c_{3}=0$. The effect of the $w_{3}$ component does not modify the results to any great extent. For example, the dotted line $k$ in Fig. 5 shows the stress-strain curve for $\beta=2$ and $w_{1}^{\prime}=1 / 32, w_{2}^{\prime}=1 / 16, c_{3}=0$. This curve does not differ substantially from the full line $e$ which corresponds to the same initial deformation, but with $w_{3}$ and Eq. (15) neglected.

(c) Third type of deflection equation

The stress and displacement functions will now be expressed in the forms

$$
\begin{aligned}
& f / t^{2}=f_{1} \sin \xi \sin \varphi+f_{\mathrm{II}} \cos \xi \sin \varphi+f_{2} \cos 2 \xi+f_{3} \cos 2 \varphi-\eta y^{2} / R t, \\
& w / t=w_{1} \sin \xi \sin \varphi+w_{\mathrm{II}} \cos \xi \sin \varphi+w_{2} \cos 2 \xi+w_{0} .
\end{aligned}
$$

The coefficients are determined from Eqs. (8) in which we write successively

$$
\psi_{1}=\psi_{2}=\sin \xi \sin \varphi,=\cos \xi \sin \varphi,=\cos 2 \xi, \quad \psi_{1}=\cos 2 \varphi .
$$

By eliminating the coefficients $f$, we obtain for $\alpha=\beta$ :

$$
\begin{aligned}
2 \eta \beta^{2}-4-\frac{\beta^{4}}{4} & =-\frac{3 \beta^{2}}{2} w_{2}+\frac{w_{1}^{2}}{8}+w_{2}^{2}+\frac{c_{1}}{w_{1}}=\frac{3 \beta^{2}}{2} w_{2}+\frac{w_{\mathrm{II}}^{2}}{8}+w_{2}^{2}+\frac{c_{\mathrm{II}}}{w_{\mathrm{II}}} \\
& =-\frac{3 \beta^{2}}{32 w_{2}}\left(w_{1}^{2}-w_{\mathrm{II}}^{2}\right)+\frac{1}{8}\left(w_{1}^{2}+w_{\mathrm{II}}^{2}\right)+\frac{c_{2}}{w_{2}} .
\end{aligned}
$$

The constants $c_{1}, c_{I I}$ and $c_{2}$ depend upon the values $w_{1}^{\prime}, w_{I I}^{\prime}$ and $w_{2}^{\prime}$ of the displacement components when $\eta=0$. 
It should be noted that the solution does not modify if the quantities $w_{1}, w_{\mathrm{II}}, w_{2}$ are respectively replaced by $w_{\mathrm{II}}, w_{1},-w_{2}$.

As before, calculations were made for $\beta=2$. For the initial displacement components $\left(w_{1}^{\prime}=1, w_{1}^{\prime}=1 / 32, w_{2}^{\prime}=-1 / 4\right)$, the stress-strain diagram is represented by tho full line in Fig. 3. Comparing this graph with line $g$, corresponding to

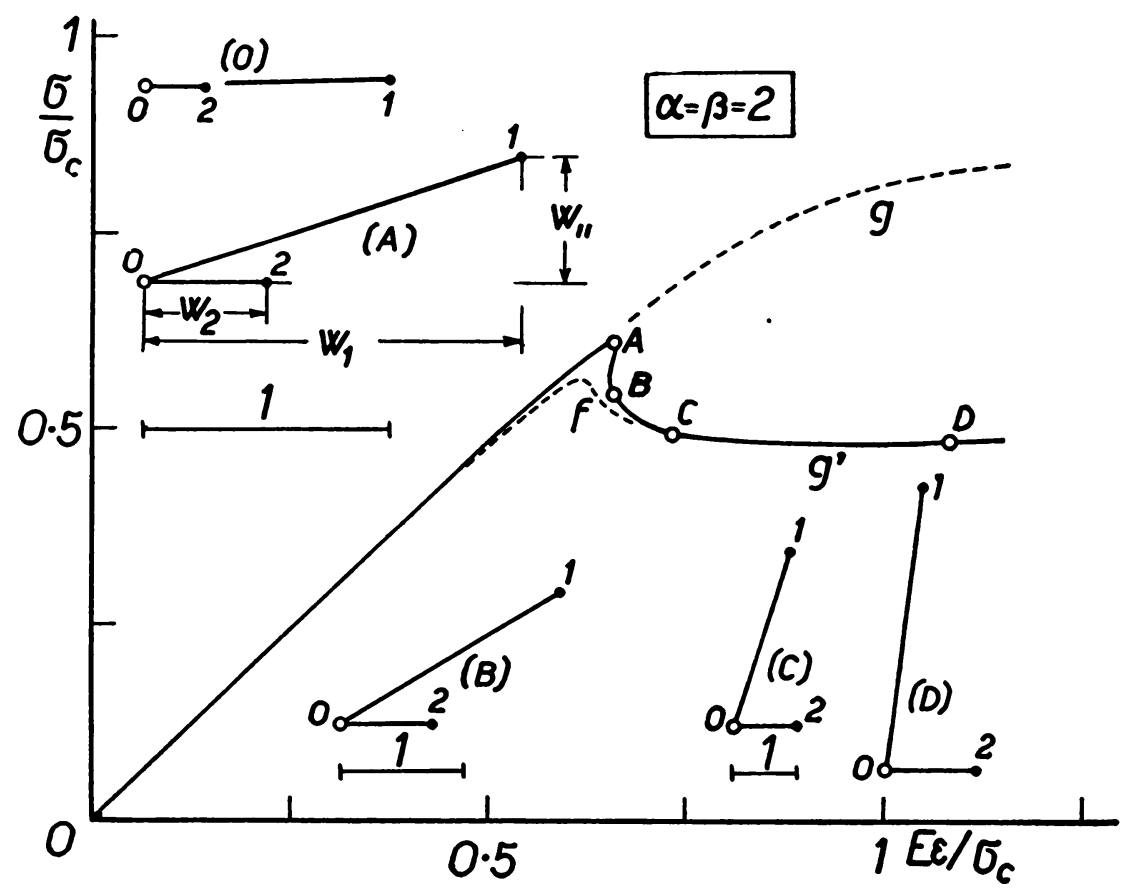

FIG. 3. Stress-strain diagram for the complete cylinder.

Line $\mathrm{f}\left(w_{1}^{\prime}=1 / 32, w_{\mathrm{II}}{ }^{\prime}=0, w_{2}^{\prime}=1 / 4\right)$, or $\left(w_{1}^{\prime}=0, w_{\mathrm{II}}{ }^{\prime}=1 / 32, w_{2}^{\prime}=-1 / 4\right)$.

Line $\mathrm{g}\left(w_{1}^{\prime}=1, w_{\mathrm{II}}{ }^{\prime}=0, w_{2}^{\prime}=-1 / 4\right)$.

Line $\mathrm{g}^{\prime}$ and vector diagrams (0), (A), (B), (C), (D) of displacement components: $\left(w_{1}^{\prime}=1, w_{\mathrm{II}}{ }^{\prime}=1 / 32\right.$, $\left.w_{2}^{\prime}=-1 / 4\right)$.

$\left(w_{1}^{\prime}=1, w_{2}^{\prime}=-1 / 4\right)$, the effect of the additional displacement term is seen to change the form from a favorable to an unfavorable one.

The evolution of the buckled form will be evident from the vector diagrams (0), (A), (B), (C) and (D) in Fig. 3, which represent the displacements for the corresponding points on the full curve. In these diagrams, the vector " 02 " represents the component $w_{2}$, the horizontal and vertical components of the vector " 01 " represent respectively $w_{1}$ and $w_{\mathrm{II}}$. Diagram (0), corresponding to $\eta=0$, indicates that $w^{\prime}$ is negligible. However, as one proceeds from (0) to (D), $w_{\mathrm{II}}$ increases rapidly, while $w_{1}$ decreases. The units for displacements are indicated on the diagrams.

Fig. 4 represents the deflections along a generator $y=0$ or $y=n b$ ( $n$ is any positive integer), for points corresponding to those on the full curve in Fig. $3 .^{3}$ The initial form

${ }^{3}$ The position of the line $w=0$ with respect to the curves in Fig. 4 is of no great interest: the value of $w_{0}$ in the expression for $w$ is determined from the condition that the mean circumferential stress is zero.

The diagrams in Fig. 4 are drawn to such a scale that the quantity $\sqrt{w_{1}^{2}+w_{\mathrm{II}}^{2}}$ is always represented by the distance apart of the horizontal lines. 
in Fig. 4-graph 0-, almost symmetrical about the verticals $x=-a / 2, a / 2,3 a / 2$, etc., corresponds to the most favorable type. This symmetry is destroyed as the load increases and, during the buckling, the form approaches the unfavorable type which has a shape similar to graph (0), but with opposite signs for the deflections.

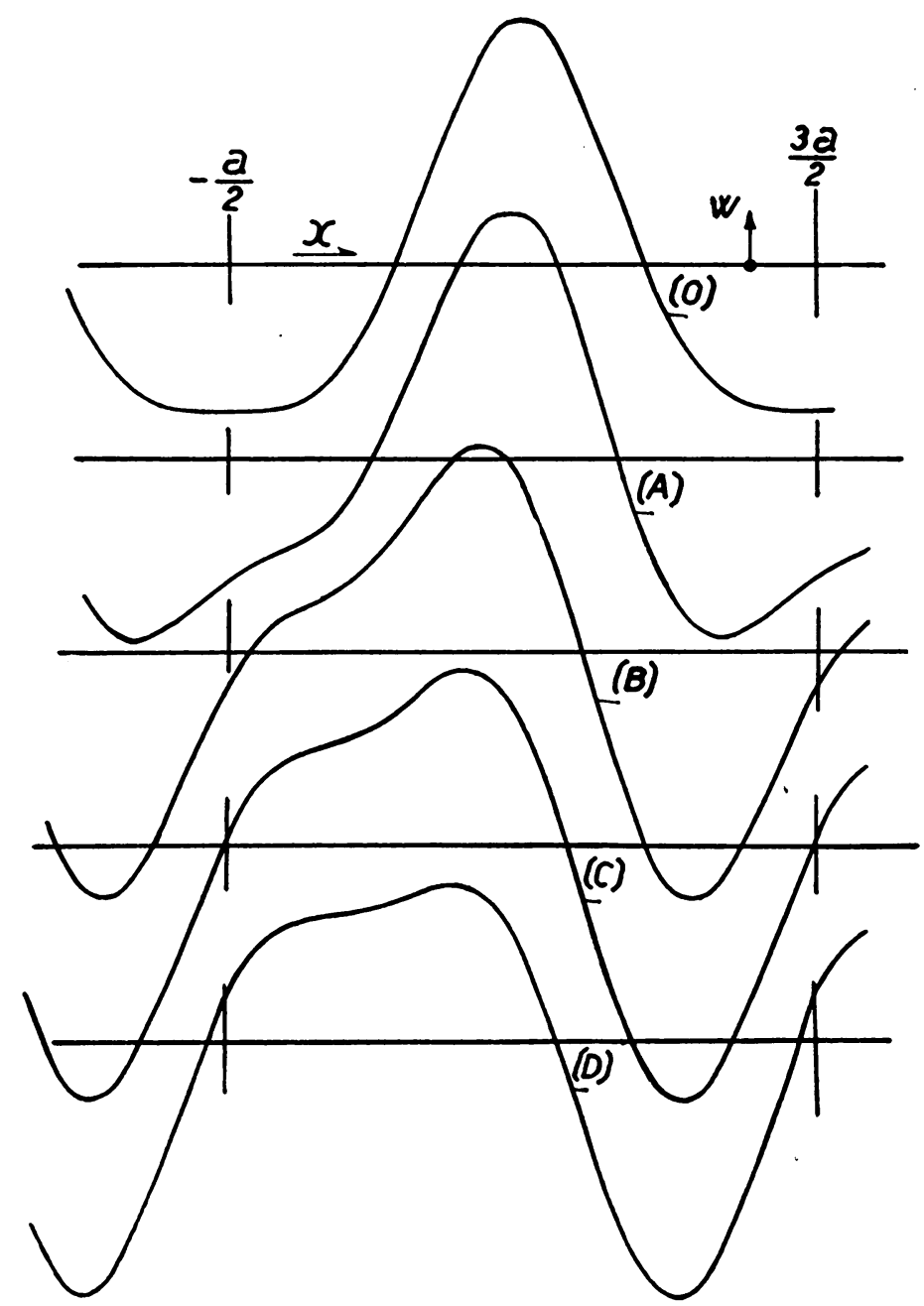

FIG. 4. Evolution of the deformation of a generator during the buckling process corresponding to line $\mathbf{g}^{\prime}$ in Fig. $3 ; w$ positive outward

Analogous results are plotted in Fig. 5. As before, the evolution of the three deformation components is represented vectorially. Starting with the initial diagram (0), for which $\left(w_{1}^{\prime}=1 / 8, w_{I I}^{\prime}=1 / 32, w_{2}^{\prime}=-1 / 16\right)$, the vector diagrams (A), (B) and (C) show, to various scales, the deformation components, in successive phases of buckling, when $-w_{2}$ has the values $1 / 8,1 / 4$ and $1 / 2$. The corresponding points in the stressstrain diagram fall approximately on curve $e$. Starting with the initial diagram $\left(0^{\prime}\right)$, for which $\left(w_{1}^{\prime}=1 / 4, w_{\mathrm{II}}^{\prime}=1 / 32, w_{2}^{\prime}=-1 / 16\right)$, in successive phases when $-w_{2}$ has 
the values $1 / 2$ and 1 , the conditions represented by diagrams (D) and (E) are attained. The corresponding points lie practically on the same curve $e$.

These results show that the behaviour of the cylinder, as calculated when the components $w_{1}, w_{\mathrm{II}}, w_{2}$ are considered, always approaches the most unfavorable path between the one determined neglecting the $w_{1}$ component and the one neglecting the $w_{\text {II }}$ component.

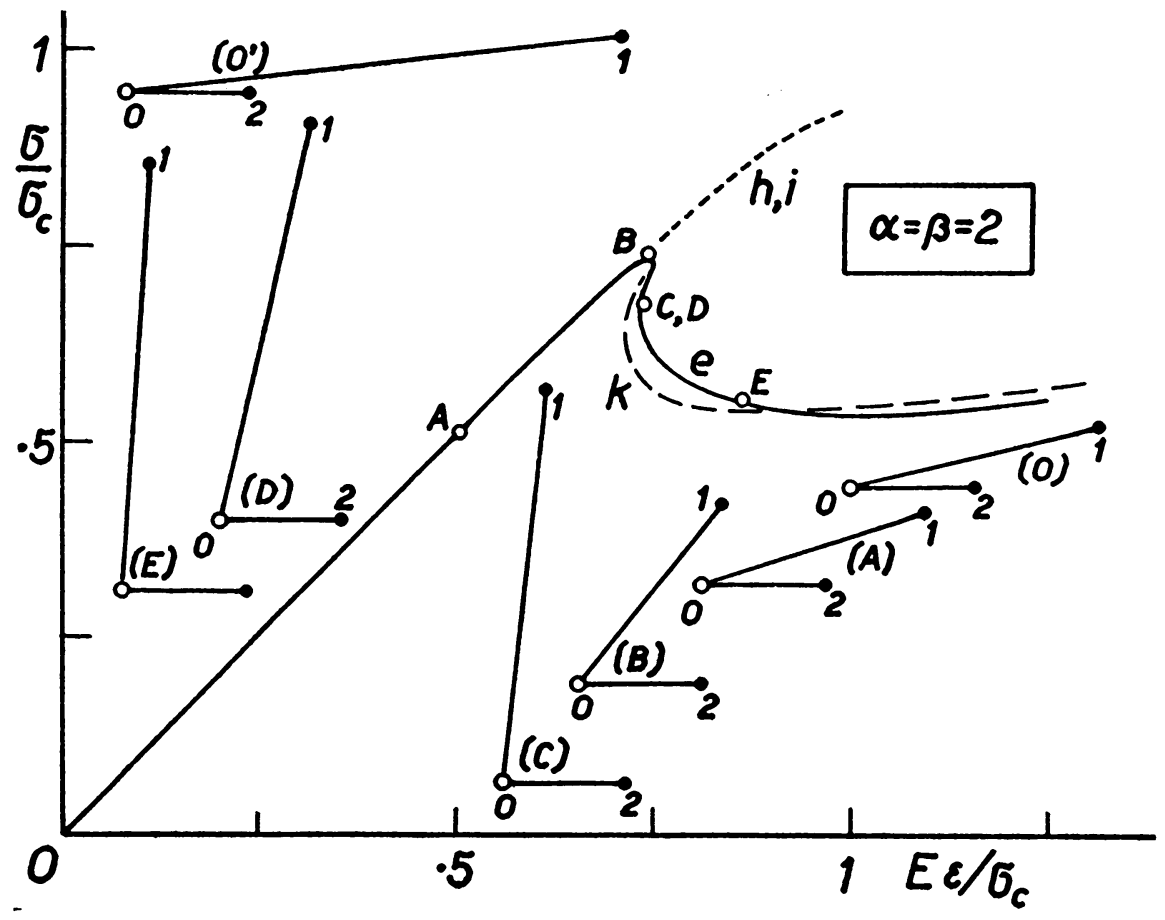

FIG. 5. Stress-strain diagram for the complete cylinder.

Line e $\left(w_{1}^{\prime}=1 / 32, w_{\mathrm{II}}{ }^{\prime}=0, w_{2}^{\prime}=1 / 16\right)$, or $\left(w_{1}{ }^{\prime}=0, w_{\mathrm{II}}{ }^{\prime}=1 / 32, w_{2}{ }^{\prime}=-1 / 16\right)$.

Line h, i $\left(w_{1}^{\prime}=1 / 8 \div 1 / 4, w_{\mathrm{II}^{\prime}}=0, w_{2}^{\prime}=-1 / 16\right)$.

Points A, B, C and vector diagrams (0), (A), (B), (C): $\left(w_{1}^{\prime}=1 / 8, w_{\mathrm{II}}{ }^{\prime}=1 / 32, w_{2}^{\prime}=-1 / 16\right)$.

Points D, E and vector diagrams $\left(0^{\prime}\right),(\mathrm{D}),(\mathrm{E}):\left(w_{1}^{\prime}=1 / 4, w_{\mathrm{II}}{ }^{\prime}=1 / 32, w_{2}^{\prime}=-1 / 16\right)$.

\section{The Curved Panel}

The problem of a curved panel which is compressed in a direction parallel to the straight sides is now considered. It is assumed that the straight sides $y=0$ and $y=b$ of the panel are simply supported and free from normal stresses and that the strain along these edges is constant (as if restrained by flexurally rigid, laterally and torsionally weak, axially very stiff side-stringers). The boundary conditions for $y=0$ and $y=b$ are therefore

$$
w=\frac{\partial^{2} w}{\partial y^{2}}=\frac{\partial^{2} f}{\partial x^{2}}=0 ; \quad \frac{\partial^{2} f}{\partial y^{2}}=-\frac{2 \eta t}{R} .
$$

The parameter $\eta$ represents here the ratio of the strain along the straight sides to the critical strain obtained from the linear theory for a complete cylinder of which the panel may be assumed to be a part. 
For the curved edges $x=0$ and $x=2 a$, we assume perfect clamping and hence

$$
w=\frac{\partial w}{\partial x}=0
$$

for these edges; in addition, we assume that these edges are free from shearing stress and write

$$
\frac{\partial^{2} f}{\partial x \partial y}=0 .
$$

The problem is considered for two extreme cases: (a) uniform longitudinal shortening and, (b) uniform longitudinal stress.

(a) Panel subjected to uniform longitudinal shortening

For the stress and displacement functions, we write respectively

$$
\begin{aligned}
f / 3 \pi t^{2} & =\left(f_{1} \cos \xi+f_{2} \cos 2 \xi+f_{3}\right) \sin \varphi-\eta y^{2} / 3 \pi R t, \\
w / 3 \pi t & =\left[w_{1}(1-\cos \xi)+w_{2}(1-\cos 2 \xi)\right] \sin \varphi,
\end{aligned}
$$

where $\xi=\pi x / a, \varphi=\pi y / b$. These functions satisfy all the boundary conditions given in Eqs. (22), (23) and (24). The condition of uniform axial shortening will be satisfied in an approximate form by writing ${ }^{4}$

$$
\int_{0}^{b}\left(\epsilon_{x}-\epsilon\right) \sin \varphi d y=0,
$$

where $-\epsilon_{x}$ is the axial shortening calculated according to Eqs. (17) and (18) and $-\epsilon$ is the edge value given by $-\epsilon=2 \eta t / R$. Thus we get

$$
\alpha^{2} f_{3} / \beta^{2}=-2 w_{1}^{2}-8 w_{2}^{2} .
$$

Furthermore, to determine the constants $f_{1}, f_{2}, f_{3}, w_{1}, w_{2}$, we use the Galerkin method and evaluate the same Eqs. (8) in which, in this case, the field of integration is defined by the panel contour and for $\psi_{1}, \psi_{2}$ we write successively the functions

$$
(1-\cos \xi) \sin \varphi, \quad(1-\cos 2 \xi) \sin \varphi .
$$

Thus, assuming $f^{\prime}=0$, we obtain

$$
\begin{aligned}
D_{1} f_{1}= & \beta^{2} w_{1}+8 w_{1}^{2}+4 w_{1} w_{2} \\
D_{2} f_{2}= & \beta^{2} w_{2}+8 w_{2}^{2}+8 w_{1} w_{2}-\frac{3}{2} w_{1}^{2} \\
2 \eta \beta^{2} w_{1}=D_{1} w_{1} & +2 \alpha^{2}\left(w_{1}+w_{2}\right) / \beta^{2}+\left(\beta^{2}+16 w_{1}-4 w_{2}\right) f_{1} \\
& +\left(32 w_{2}-12 w_{1}\right) f_{2}-8 w_{1} f_{3}+c_{1},
\end{aligned}
$$$$
2 \eta \beta^{2} w_{2}=D_{2} w_{2}+\alpha^{2}\left(w_{1}+w_{2}\right) / 2 \beta^{2}+\left(\beta^{2}+16 w_{2}+8 w_{1}\right) f_{2}-w_{1} f_{1}-8 w_{2} f_{3}+c_{2},
$$

${ }^{4}$ By substituting an arbitrary function of $\varphi$ instead of $f_{3} \sin \varphi$ in the expression for $f$ and using $\epsilon_{x}=\epsilon$ to find the function, it is possible to satisfy exactly the condition that the shortening of all generators is constant. 
where

$$
D_{1}=\left(\frac{\alpha}{\beta}+\frac{\beta}{\alpha}\right)^{2}, \quad D_{2}=\left(\frac{\alpha}{2 \beta}+\frac{2 \beta}{\alpha}\right)^{2} .
$$

By elimination of $f_{1}, f_{2}$ and $f_{3}$, from Eqs. (27) and (28) we obtain two equations in $w_{1}, w_{2}$ and $\eta$, and hence a fourth degree equation in $w_{1}$ and $w_{2}$. This furnishes corresponding pairs of $w_{1}$ and $w_{2}$ and these may be used to determine the ratio $\eta$ and the axial shortening.

The load sustained by the panel, expressed by

$$
N=t^{\prime} \int_{0}^{b} \sigma_{x} d y
$$

varies with the section considered. We therefore define an equivalent mean stress $\sigma$ as the stress which, when multiplied by the mean shortening and the volume of the panel,

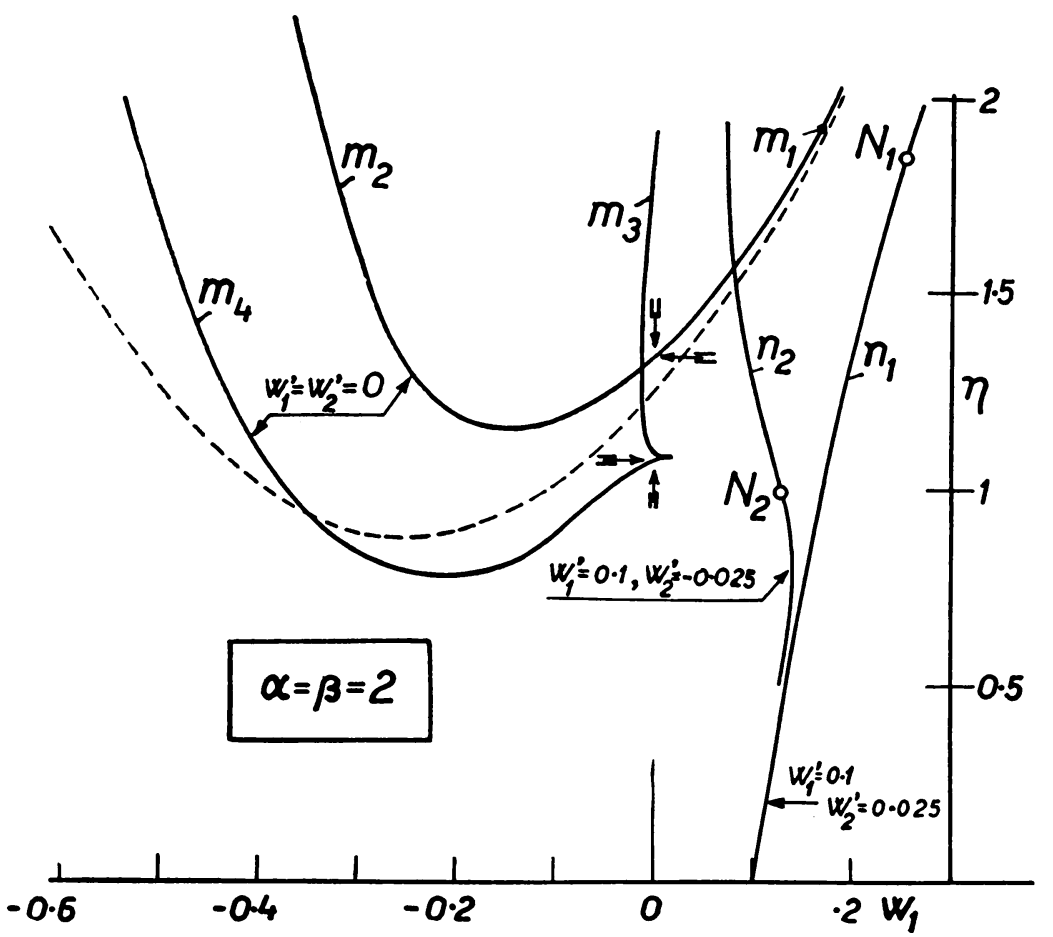

Frg. 6. Panel subjected to uniform shortening. Shortening parameter $\eta$ versus displacement component $w_{1}$.

represents the virtual work done by the forces applied to the panel for the displacements corresponding to the actual deformation. It may be shown that, according to the condition of uniform shortening, this gives

and finally

$$
\sigma=\frac{1}{2 a b t^{\prime}} \int_{0}^{2 a} N d x
$$

$$
\sigma / \sigma_{c}=\eta+3 f_{3} / \beta^{2}
$$




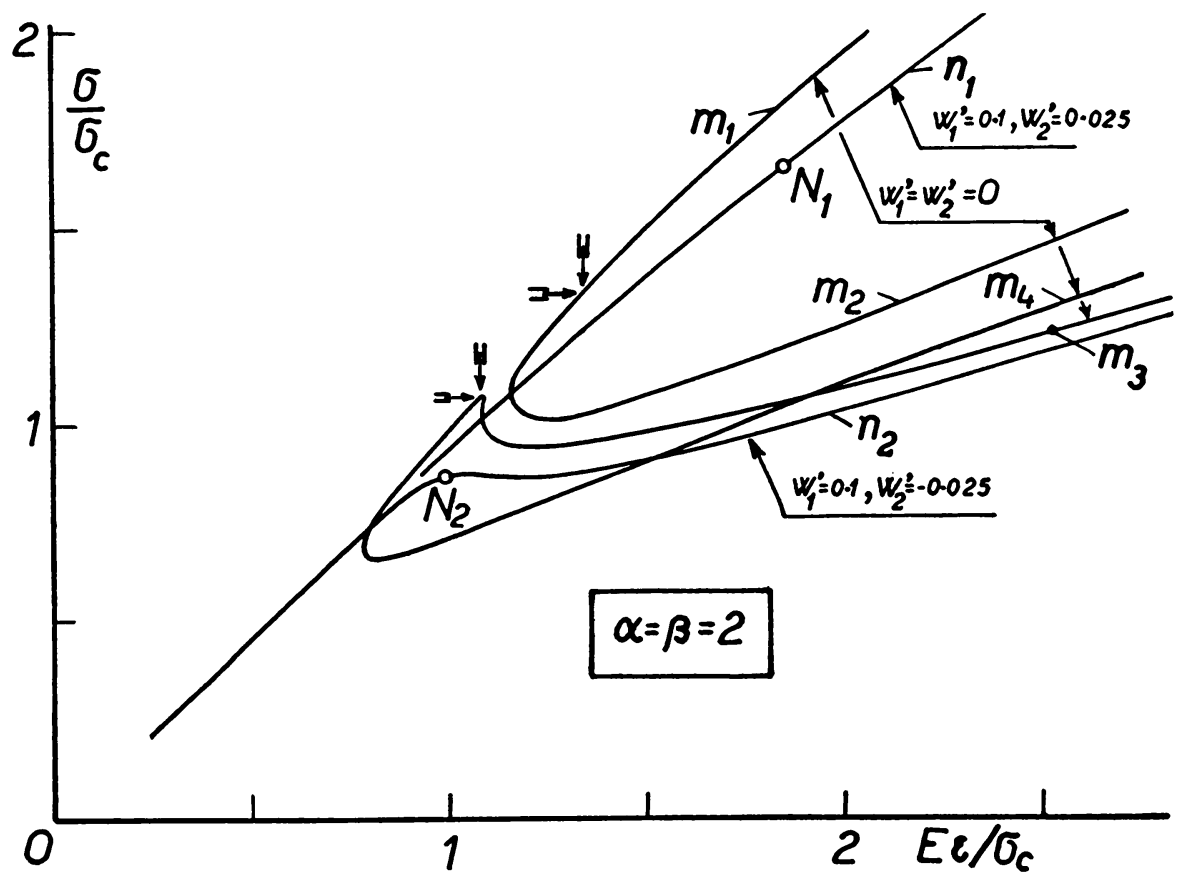

Fig. 7. Panel subjected to uniform shortening. Stress-strain diagram.

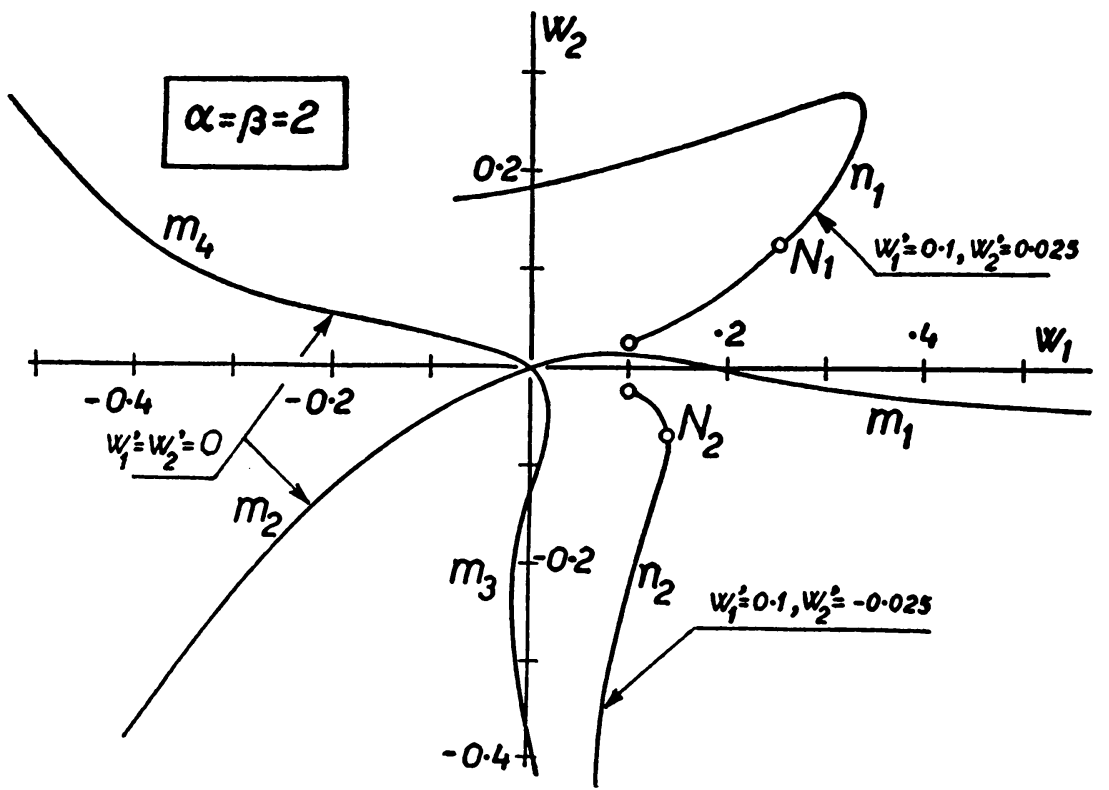

Fig. 8. Panel subjected to uniform shortening. Displacement components $w_{2}$ versus $w_{1}$.

Numerical calculations were made for $\alpha=\beta=2$ and the results are plotted in Figs. 6, 7 and 8; corresponding points and lines in the figures are marked with the same letters.

The curves $m_{1} m_{2}$ and $m_{3} m_{4}$ in the figures apply to a panel with perfect initial form, and the arrows on the curves in Figs. 6 and 7 denote points where the deflection components $w_{1}$ and $w_{2}$ are both zero. Of the four branches of the curves starting from these 
points, only $m_{1}$ gives a favorable characteristic. Favorable behaviour of the panel can only be expected for initial deformations whose representative points fall on or near the branch $m_{1}$ in Fig. 8. This indicates that favorable initial deformations are directed outwards, with maximum values near the center of the panel.

Curves $n_{1}$ and $n_{2}$ depart from the favorable path $m_{1}$ and this phenomenon is similar to that observed for a complete shell. Curve $n_{2}$ departs immediately towards the large deflection regime with little increase in the end load. However, the initial part of the

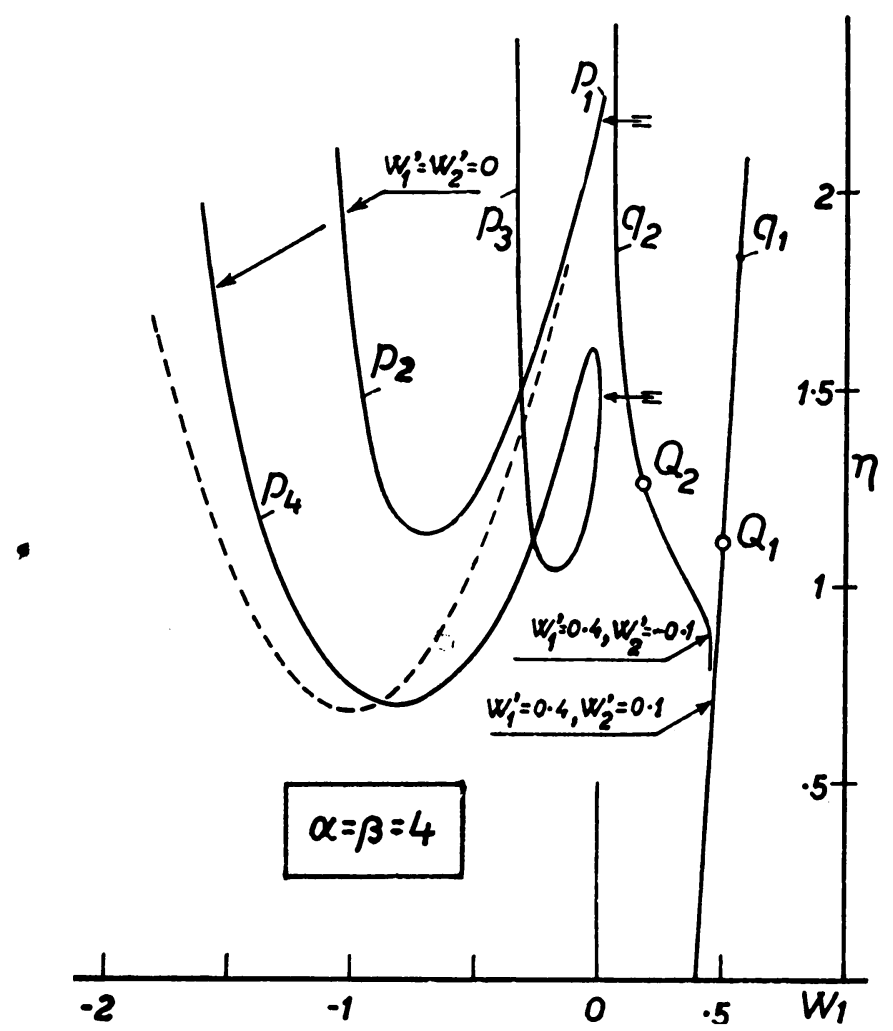

FIG. 9. Panel subjected to uniform shortening. Shortening parameter $\eta$ versus displacement component $w_{1}$.

$n_{1}$ curve corresponds to increasing loads, as shown in Fig. 7. After reaching a certain point, the curve in Fig. 8 suddenly veers in the direction of the $m_{4}$ branch. This phase occurs with decrease of load but, before it occurs, the load reaches such large values that stresses may be obtained in excess of the elastic limit of the material. For this reason, the portion of the $n_{1}$ curve in Fig. 7 which corresponds to decreasing loads is not shown.

Similar results were obtained from calculations made for $\alpha=\beta=4$, the various graphs being given in Figs. 9, 10 and 11. From the points corresponding to $w_{1}=w_{2}=0$, which are indicated by arrows in Figs. 9 and 10, the favorable path is $p_{1}$. Line $q_{1}$ suddenly veers towards $p_{4}$ but, before it does so, very high loads are attained. Line $q_{2}$ soon approaches the branch $p_{3}$ in Fig. 11 and indicates unfavorable initial deformations.

The dotted curves in Figs. 6 and 9 correspond to $w_{2}=f_{2}=0$, when the second and fourth of Eqs. (28) are left out. 


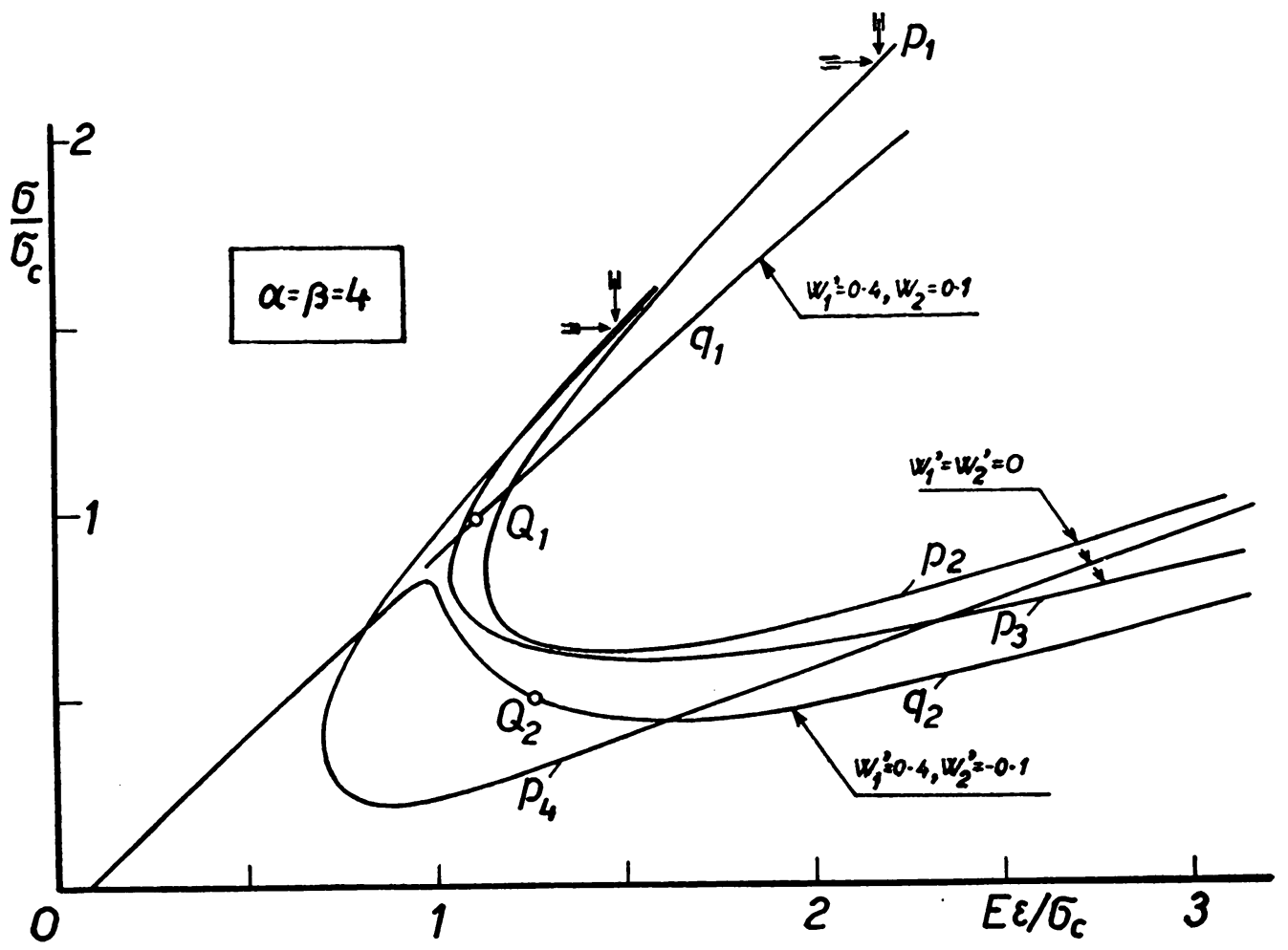

FIG. 10. Panel subjected to uniform shortening. Stress-strain diagram.

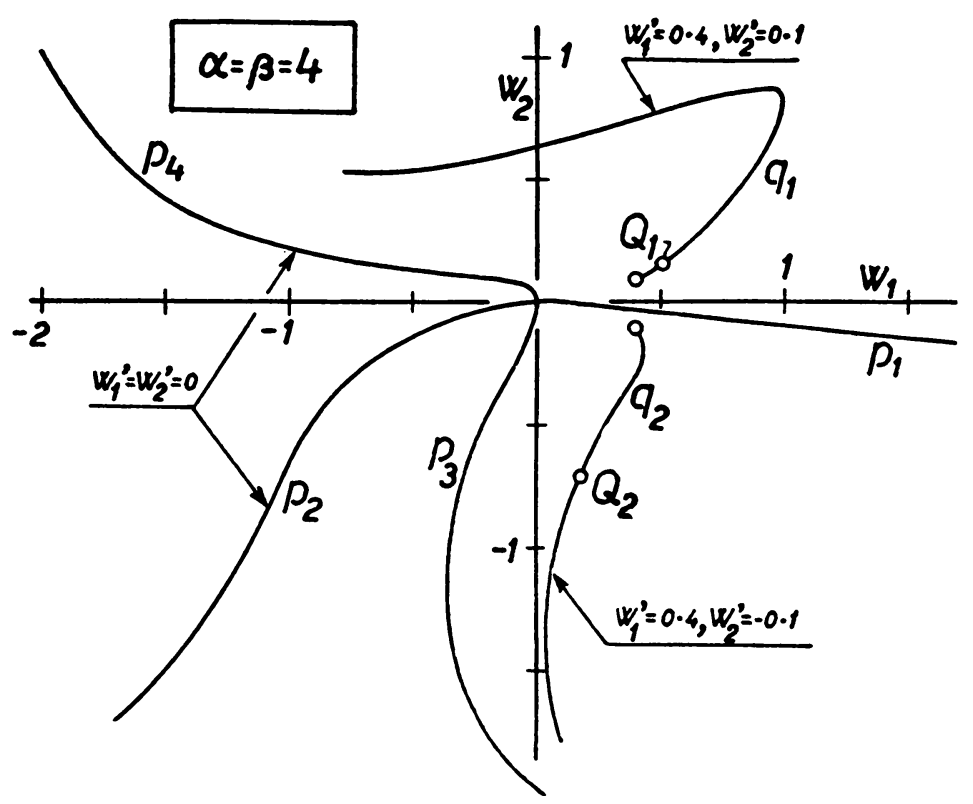

Fig. 11. Panel subjected to uniform shortening. Displacement components $w_{2}$ versus $u_{1}$. 
(b) Panel subjected to uniform longitudinal stress

The new boundary condition, replacing the one expressed by Eq. (26), is

$$
\frac{\partial^{2} f}{\partial y^{2}}=-2 \eta t / R \quad \text { for } \quad x=0 \quad \text { and } \quad x=2 a .
$$

The parameter $\eta$ represents here the ratio of the applied uniform stress to the critical stress obtained from the linear theory for a complete cylindrical shell. The new con-

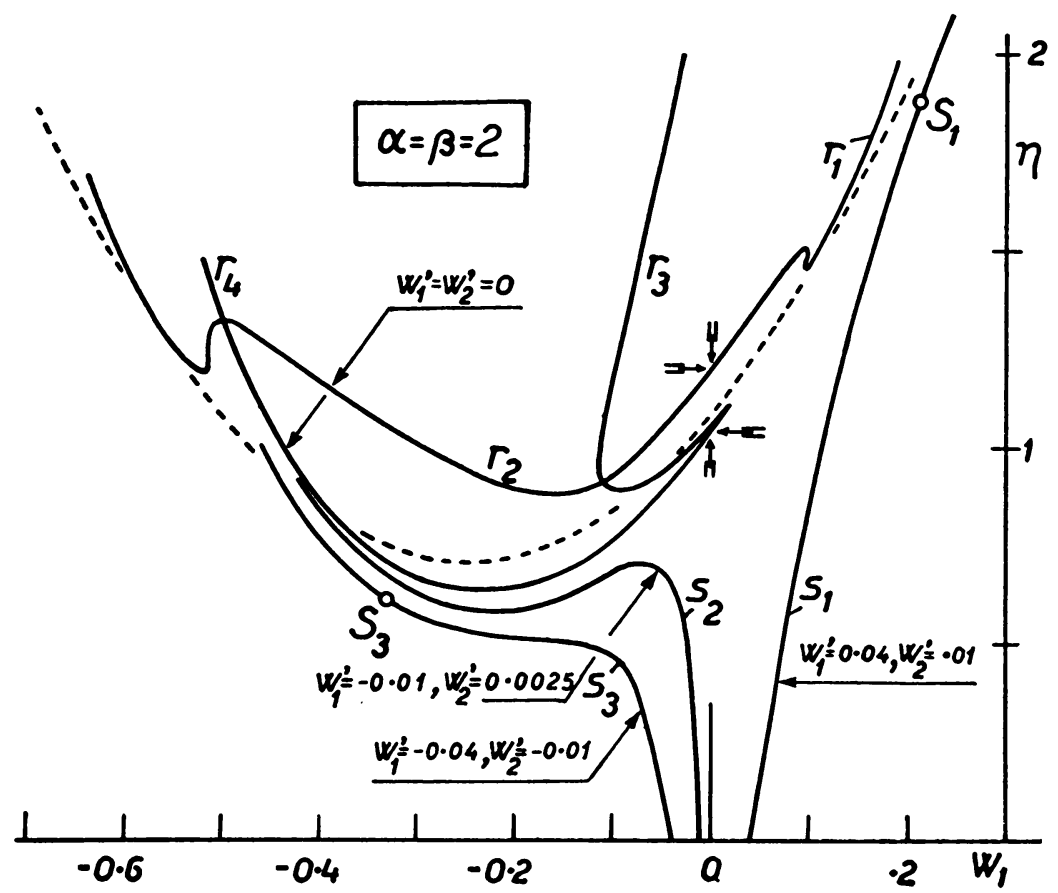

FIG. 12. Panel subjected to uniform longitudinal stress. Stress parameter $\eta$ versus displacement component $w_{1}$.

dition, as well as those expressed by Eqs. (22), (23) and (24) are found to be satisfied by writing

$$
\begin{aligned}
& f / 3 \pi t^{2}=\left[f_{1}(1-\cos \xi)+f_{2}(1-\cos 2 \xi)\right] \sin \varphi-\eta y^{2} / 3 \pi R t, \\
& w / 3 \pi t=\left[w_{1}(1-\cos \xi)+w_{2}(1-\cos 2 \xi)\right] \sin \varphi .
\end{aligned}
$$

Proceeding as before, we obtain

$$
\begin{aligned}
& D_{1} f_{1}+2 \frac{\alpha^{2}}{\beta^{2}}\left(f_{1}+f_{2}\right)=-\beta^{2} w_{1}-12 w_{1}^{2}-16 w_{2}^{2}+4 w_{1} w_{2} \\
& D_{2} f_{2}+\frac{\alpha^{2}}{2 \beta^{2}}\left(f_{1}+f_{2}\right)=-\beta^{2} w_{2}-12 w_{2}^{2}-\frac{1}{2} w_{1}^{2}-8 w_{1} w_{2}
\end{aligned}
$$


$2 \eta \beta^{2} w_{1}=D_{1} w_{1}+2 \frac{\alpha^{2}}{\beta^{2}}\left(w_{1}+w_{2}\right)-\left(\beta^{2}+24 w_{1}-4 w_{2}\right) f_{1}-\left(32 w_{2}-4 w_{1}\right) f_{2}+c_{1}$, $2 \eta \beta^{2} w_{2}=D_{2} w_{2}+\frac{\alpha^{2}}{2 \beta^{2}}\left(w_{1}+w_{2}\right)-\left(\beta^{2}+24 w_{2}+8 w_{1}\right) f_{2}-\left(8 w_{2}-w_{1}\right) f_{1}+c_{2}$, with the same notation previously used.

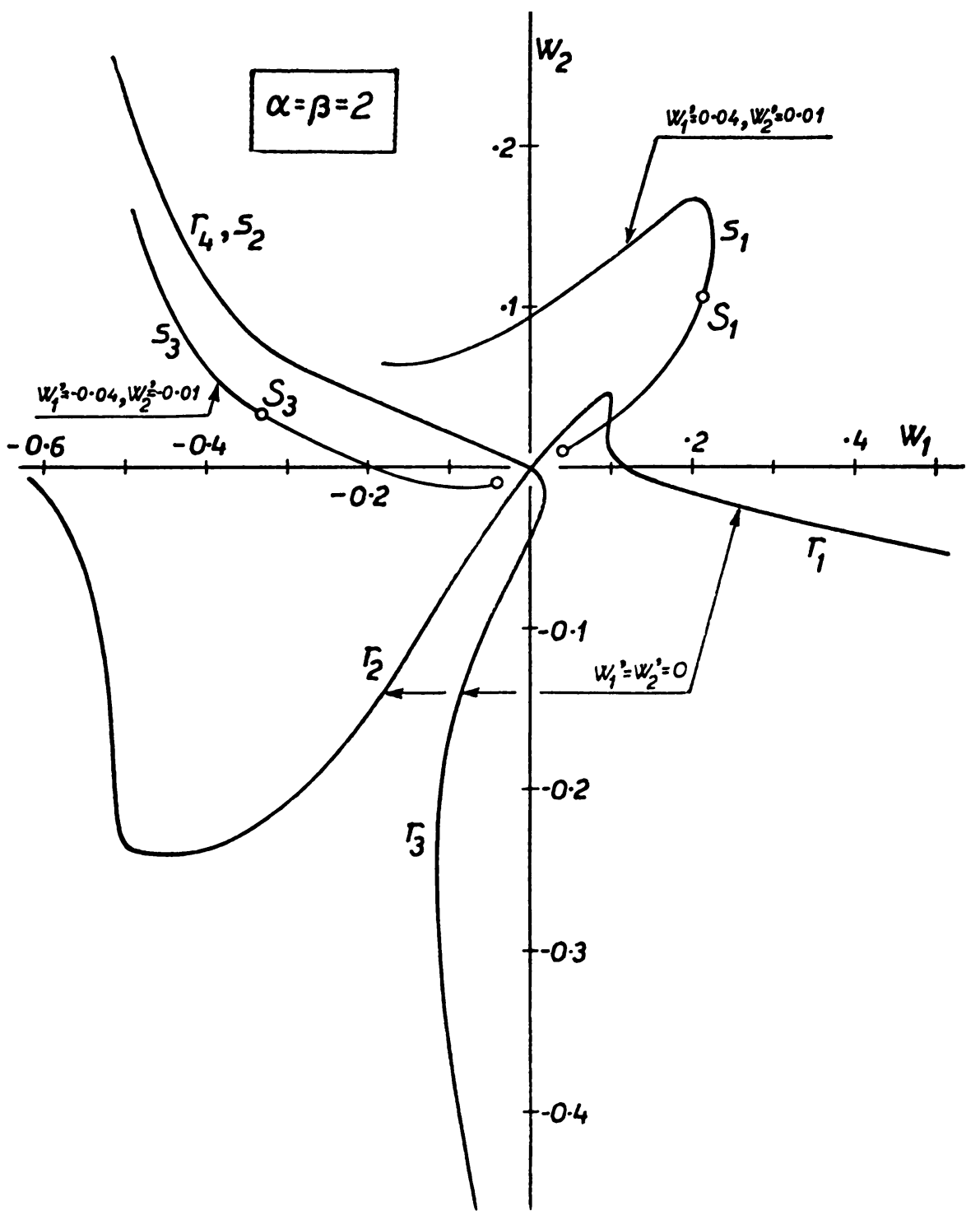

FIG. 13. Panel subjected to uniform longitudinal stress. Displacement components $w_{2}$ versus $w_{1}$.

Calculations were made for $\alpha=\beta=2$ and the results plotted in Figs. 12 and 13 . Stress-strain diagrams were not plotted since the end displacement varies with the particular generator chosen. 
The conclusions drawn for a panel with uniform shortening also apply to a panel with uniform longitudinal stress. The arrows in Fig. 12 show the points corresponding to zero values of $w_{1}$ and $w_{2}$ for a panel without initial deformations. Branches $r_{1}, r_{2}$, $r_{3}$ and $r_{4}$ starting from these points correspond to the branches starting from the origin in Fig. 13.

Curves $r_{4}$ and $s_{2}$ in Fig. 13 are practically coincident. The dotted curve in Fig. 12 is obtained equating $w_{2}$ and $f_{2}$ to zero and disregarding the second and fourth of Eqs. (31).

\section{Conclusions}

(a) The cylindrical shell under axial compression

An approximate analysis has been made of the behaviour of an axially loaded cylindrical shell using a four-term expression for the stress function and also for the displacement function. However, numerical calculations were generally made using three terms in

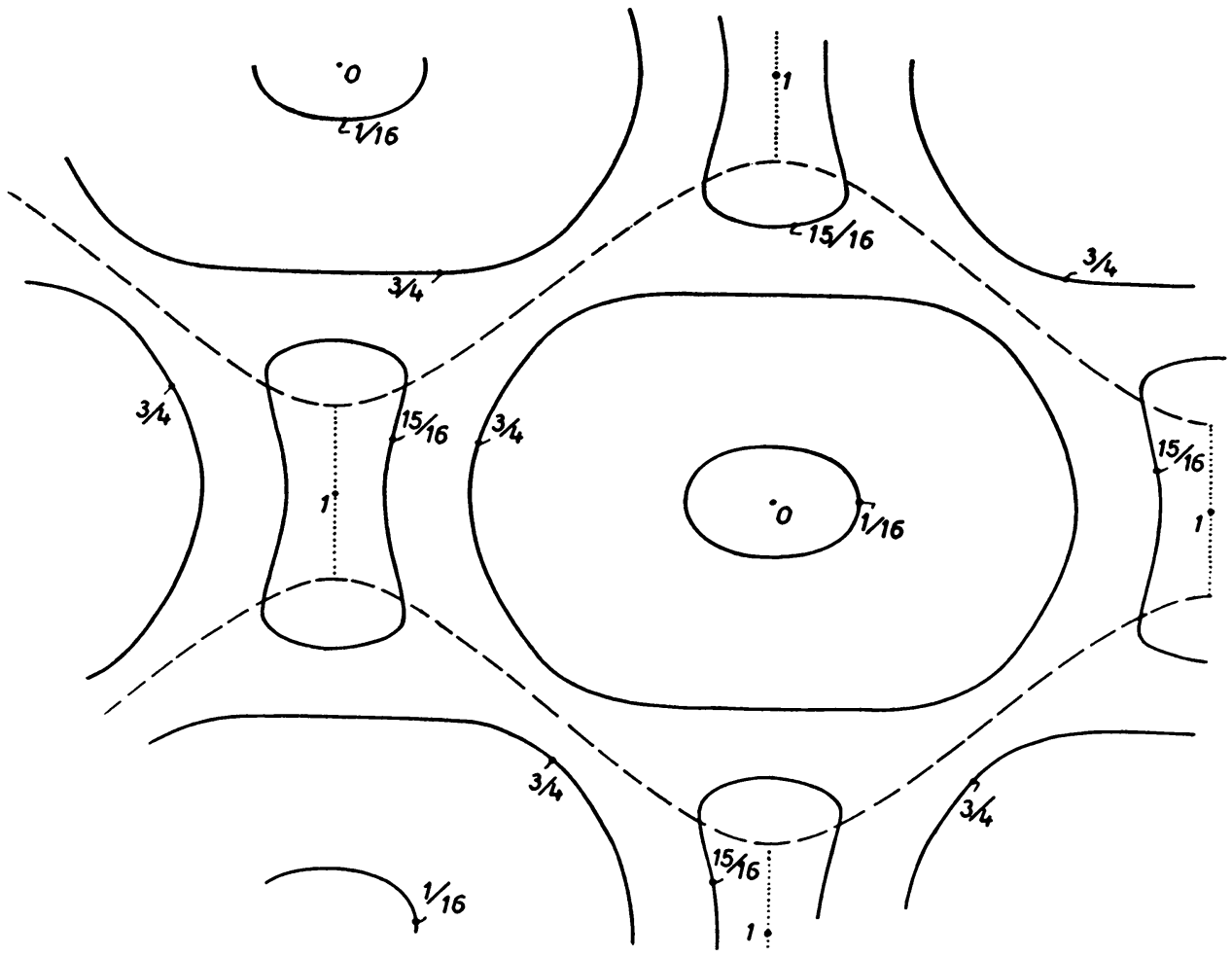

FIG. 14. Lines of equal deflection of wave surfaces, according to eq. (10), for $w_{3}=w_{4}=0, w_{1}=3.2 w_{2}$.

the expressions for the stress functions and two in the expressions for the displacements. The two terms retained are of the form $\sin (\pi x / a) \sin (\pi y / b)$ and $\cos (2 \pi x / a)$, since previous experience has shown (2) that these terms are of greatest importance in the first phase of the buckling process.

Three modes of buckling appear possible for a cylinder with perfect initial form. 
The most unfavorable one corresponds to the lower branch $\mathrm{OB}$ of the corresponding curve in Fig. 1. In this case, the ratio of the amplitudes of the displacement components is about 4 . The generators of the cylinder which contain the crests of the waves in the buckled form take a shape similar to that given by the curve (0) in Fig. 4 but with opposite sign, that is, with flat tops directed outwards from the cylinder and sharp tops directed inwards.

The curves formed by the intersection of the deflected surface with coaxial cylinders of various radii are shown in Fig. $14 ;^{5}$ they refer to the case when the ratio of the amplitudes is 3.2. The axis of the cylinder is vertical in Fig. 14 and it is evident that the outwardly directed crests are elongated in the direction of the axis. This phenomenon is often noticed in buckled specimens.

The favorable type of buckling whose stress-strain characteristics are represented by the branch $0 \mathrm{~A}$ of the curve in Fig. 1, has generators of the form given by curve (0) in Fig. 4. If this type of deformation could be ensured, the behaviour of a cylindrical shell under axial load would be practically unaffected by buckling, and the stress-strain curve would continue to follow the line representing Hooke's law.

The third type of buckling corresponds to an axially symmetrical configuration and this would be represented on the stress-strain diagram in Fig. 1 as a horizontal line through the point 0 .

When the second deflection component in Eq. (7) is initially positive (that is, the inward crests are sharper than the outward ones), under axial loading, the deformations rapidly approach the most unfavorable form, no matter what the magnitudes of the initial deformations may be. The stress-strain curves for various initial deformations are given in Fig. 1.

When the second component in Eq. (7) is initially negative, it might seem possible to produce a favorable mode of buckling. However, a more accurate analysis has shown this to be impossible. If the first component is larger than the second, the magnitude of the latter is further decreased by axial loading until it reverses its sign and gives rise to the unfavorable mode of buckling. This is indicated by the curves $c$ and $d$ in Fig. 2 . If the first component is the smaller, the second component could increase under load and lead to axially symmetrical buckling. However, this could only occur if planes normal to the axis of the cylinder containing the maxima of the first component, also contained the maxima of the second component. In reality this perfect coincidence never occurs. Then, as is shown by the analysis based on Eqs. (20), a rapid relative shift of the two components occurs. When the position of one component has changed by half a wavelength with respect to the other, the deflected form is practically inverted as indicated by the various curves in Fig. 4 . The stress-strain curve for this case is still unfavorable.

It may therefore be concluded that, irrespective of the initial deformations existing in a cylindrical shell, buckling will always follow an unfavorable mode, with the stressstrain curve rapidly approaching that pertaining to the lower branch for a shell of perfect initial form. With cylinders having initial deformations of wavelength about $2 \pi \sqrt{R t}$, it is impossible to improve the stress-strain characteristics.

${ }^{5}$ The radial distance from the intersection to the internally tangent cylinder is given on each curve as a fraction of the difference in radii of the externally and internally tangent cylinders. The dotted curve is the locus of points where the tangent plane is parallel to the axis of the cylinder: 
For wavelengths greater than $2 \pi \sqrt{R t}$, it may be shown that, at buckling, the stressstrain curves start from a point for which $\eta>1$ but that they fall rapidly to low minima. Such initial deformations are therefore dangerous.

The possibility of using initial deformations to improve the stress-strain characteristics of cylinders is only feasible for wavelengths considerably smaller than $2 \pi \sqrt{R t}$. Such wavelengths might stiffen the shell and discourage the buckling. This possibility is best investigated experimentally. ${ }^{6}$

(b) The panel under axial compression

The compressed cylindrical panel was analyzed assuming, as to radial displacements, that the curved sides were clamped and that the straight sides were simply supported. The expression chosen for the radial displacements contains two terms and Fig. 15
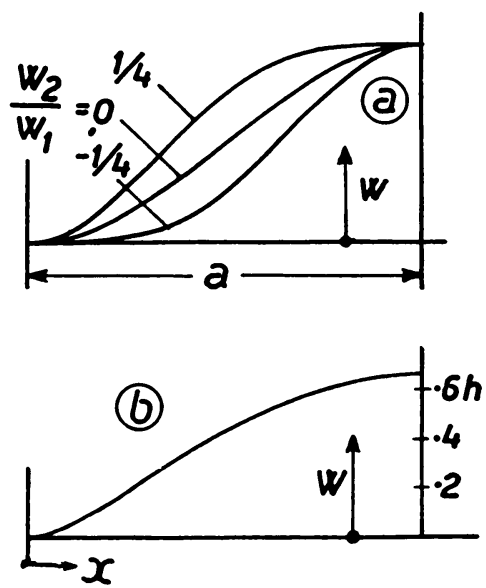

Fig. 15. Deformation of mid-generator, for $a=b$; (a) according to eq. (32), (b) according to eq. (45).

shows the forms assumed by the middle generator for three ratios of the maximum values of these terms.

Calculations were made for two special cases: (a) panel subjected to constant axial shortening and, (b) panel subjected to constant axial stress. The conclusions drawn from the two cases are the same. As for a complete cylindrical shell, the deformations tend towards the unfavorable modes but it is possible for the critical load of the linear theory to be exceeded before the beneficial effects of certain initial deformations are nullified. The approximate analysis given in the Appendix shows that favorable initial deformations may be obtained by initially bending a panel to a radius which is smaller than that at which it is tested. This may be seen from the stress-strain curves $n_{1}$ and $q_{1}$ in Figs. 7 and 11 respectively, which correspond to the curve $w_{2} / w_{1}=1 / 4$ in Fig. 15(a); Welter's experimental results also indicate that a continuous and regular stressstrain curve is possible. With proper experimental verification, the foregoing analysis will serve to indicate how the behaviour of a compressed panel may be improved.

${ }^{6}$ For short cylinders, the barrel shape might be beneficial. 


\section{ACKNowledgement}

This investigation was carried out as a part of the Cornell Aeronautical Laboratory program of internal research, and the author wishes to express his appreciation to Dr. A. F. Donovan, Head of the Aero-Mechanics Department of the Laboratory who suggested this problem for investigation. The author is greatly indebted to Prof. H. D. Conway of Cornell University for his accurate revision and linguistic correction of the paper.

\section{BIBLIOGRAPHY}

1. Th. von Kármán and H. S. Tsien, The buckling of thin cylindrical shells under axial compression, J. Aero. Sci., 8, 303-312, (1941).

2. P. Cicala, Il cilindro in parete sottile compresso assialmente. Nuovo orientamento dell'indagine sulla stabilità elastica, L'Aerotecnica, XXIV, 3-18, (1944).

3. G. Welter, The effect of radius of curvature and preliminary artificial eccentricities on buckling loads of curved thin aluminum-alloy sheets for monocoque constructions, J. Aero. Sci., 13, 593-596, (1946).

4. S. Dei Poli, Sul calcolo delle pareti cilindriche compresse secondo le generatrici, Politecnico di Milano, June, 1946.

\section{APPENDIX}

Initial deformations caused by clamping a panel in cylindrical grips.

In Welter's tests (3) some panels were bent to various radii and then tested in grips of smaller radius. In order to estimate the initial deformations likely to have occurred in those conditions, we consider the following schematic problem.

A rectangular panel whose sides are $x=0, x=2 a, y=0$ and $y=b$, is initially bent to a shape $w^{*}=h \sin \varphi$ and is then constrained such that the following boundary conditions are imposed

$$
w=\frac{\partial w}{\partial x}=0 \quad \text { for } \quad x=0,2 a ; \quad w=\frac{\partial^{2} w}{\partial y^{2}}=0 \quad \text { for } \quad y=0, b .
$$

Making use of Eq. (5) and assuming small displacements, we write

$$
\nabla^{4}\left(w-w^{*}\right)=0 .
$$

According to the boundary conditions, the solution takes the form:

$$
\begin{gathered}
w=\frac{h \sin \varphi}{\sinh \chi_{0} \cosh \chi_{0}+\chi_{0}}\left[\left(\sinh \chi_{0}+\chi_{0} \cosh \chi_{0}\right)\left(\cosh \chi_{0}-\cosh \chi\right)\right. \\
\left.\quad+\left(\chi \sinh \chi-\chi_{0} \sinh \chi_{0}\right) \sinh \chi_{0}\right]
\end{gathered}
$$

where

$$
\chi=\pi(x-a) / b, \quad \chi_{0}=\pi a / b .
$$

This equation gives an estimate of the initial deformations occurring when a panel, initially bent to a cylindrical surface of small curvature, is clamped in grips, also of 
small curvature, but with a camber different by $h$ from that of the panel. The deflections obtained for $a=b$, for the generator $y=b / 2$ are plotted in Fig. 15(b).

The condition encountered in Welter's tests were, of course, somewhat different from the above. For example, the grips in the tests gave a large camber to the panels and this would require the use of Eqs. (5) in their complete form. Rotation of the straight edges was also restricted by the toothed guides. For these and other reasons, it is obvious that the above is not a rigorous solution. However it will give an estimate of the initial deformations which may be expected. 